\title{
A Dual-Mode Millimeter-Wave Folded Microstrip Reflectarray Antenna
}

\author{
I-Young Tarn, Yu-Shin Wang, and Shyh-Jong Chung, Senior Member, IEEE
}

\begin{abstract}
A dual-mode folded microstrip reflectarray antenna was developed and demonstrated in this paper. The proposed folded reflectarray antenna contains three parts: a planar main reflector, a planar subreflector, and printed feed antennas. The main reflector is used to produce twisted reradiated fields and to provide phase compensation for focusing. The subreflector parallel with the main reflector is made of a substrate printed with high-density metal grid lines, which is transparent to perpendicularly polarized fields, but would reflect the parallel ones. Three fixed-position patch antennas with polarization parallel to the grid lines are created for the radar mode, so that the radiation beam is switchable. Another patch with perpendicular polarization is designed for communication. A simple approach was proposed for simulating and designing the folded reflectarray. Measured results show good agreement with the calculated ones.
\end{abstract}

Index Terms-Beam switching, dual-mode, folded reflectarray, millimeter wave.

\section{INTRODUCTION}

$\mathbf{T}$ HE reflectarray antenna [1]-[10] presented in this paper can be simultaneous operated in two modes, namely, the radar mode and the communication mode. In the radar mode, the antenna demonstrates high gain and narrow beamwidth characteristics with beam switching capability. It is suitable for radar applications, such as automotive sensors in wireless Intelligent Transport Systems (ITS). While in the communication mode, the beamwidth is much broader and thus provides communications over a wide angular range.

The radar is a key technology in ITS, which opens up new perspectives of comfort and safety features in future automobiles. The automotive radar can be used for target identification, road condition detection, vehicle collision warning and avoidance, obstacle warning, stop-and-go traffic support and cruise control. In addition, accurate radar images about the ambient traffic situation could be applied for multiple targets classification and scenario interpretation. For a forward looking vehicle radar, a coverage of about \pm 10 degree, deduced from the demand of operation in urban area or narrow road curvature, is acceptable. The required field of view can be achieved using a multibeam or steerable beam high-resolution antenna. In general, a narrower

Manuscript received January 29, 2007; revised October 21, 2007. This work was supported by the National Science Council under Grant 96-XXXX.

I-Y. Tarn is with the Department of Communication Engineering, National Chiao Tung University, Hsinchu, Taiwan 300, R.O.C. and also with the Electrical Engineering Division, National Space Organization (NSPO), Hsinchu 30077, Taiwan, R.O.C. (e-mail: tarn.cm89g@nctu.edu.tw).

Y.-S. Wang and S.-J. Chung are with the Department of Communication Engineering, National Chiao Tung University, Hsinchu, Taiwan 300, R.O.C. (e-mail: elesiin.cm88@nctu.edu.tw; sjchung@cm.nctu.edu.tw).

Digital Object Identifier 10.1109/TAP.2008.923310

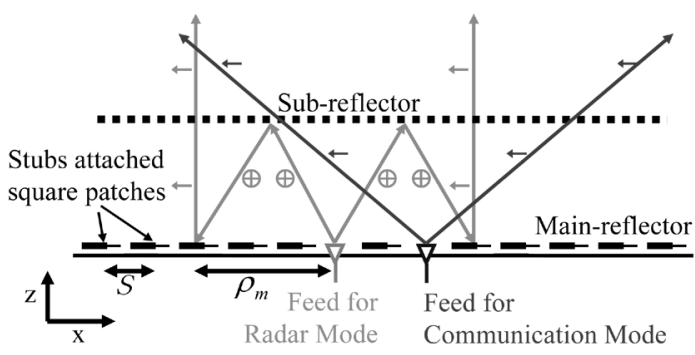

Fig. 1. The schematic diagram of the proposed folded reflectarray antenna.

beamwidth antenna is required to obtain higher resolution, and higher gains contribute considerably to the sensitivity.

Besides, there is an increasing trend in having a combined use of the inter-vehicle communications as well as the radar sensing. The inter-vehicle communications play an essential role in an advanced ITS for it enables each vehicle to communicate with other vehicles (not only directly, but also indirectly via roadside communication units), so as to get the information that are difficult or impossible to measure by the vehicle alone. The vehicular collision avoidance capability can be enhanced by incorporating inter-vehicle communications technology through wireless ad hoc networks.

The design of each constituting part of the proposed antenna is presented in this paper. Some formulas have been derived for analysis and design. Measurement results of the antenna pattern, antenna gain, aperture efficiency, and beam switching property show good agreement with the simulated ones.

\section{PRINCIPLE}

The proposed dual-mode folded reflectarray antenna is illustrated in Fig. 1. The distance $H$ from the main reflector to the subreflector is $23 \mathrm{~mm}$. The main reflector is a reflectarray that consists of hundreds of square patch antennas distributed over a circular region with diameter $D$; each square patch has two open microstrip stubs for field twisting and phase compensation. The subreflector is composed of high-density printed metal lines, which is transparent to one polarization but would reflect the other. The feed antennas are probe-fed rectangular patch antennas located on the main reflector.

For the radar mode, the fields radiated from the feed antennas are polarized in the direction parallel to the metal grid lines on the subreflector. The fields confront the subreflector are reflected. Due to the path differences, the fields received by every square patch antenna on the main reflector have different phases and amplitudes. Each square patch has two open stubs to provide the required $90^{\circ}$ polarization twisting and phase compensation. The fields reradiated from the square patch array are in uniform 


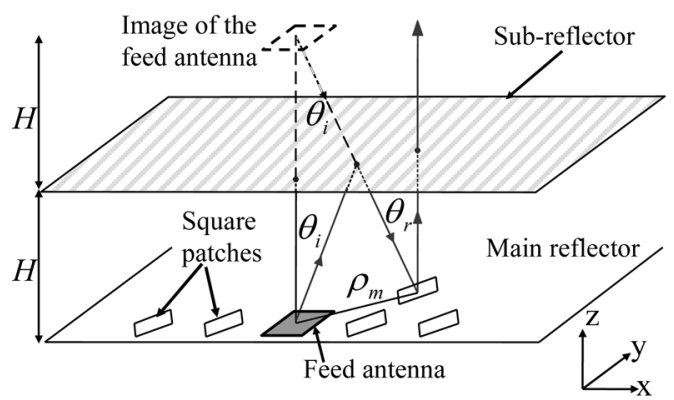

(a)

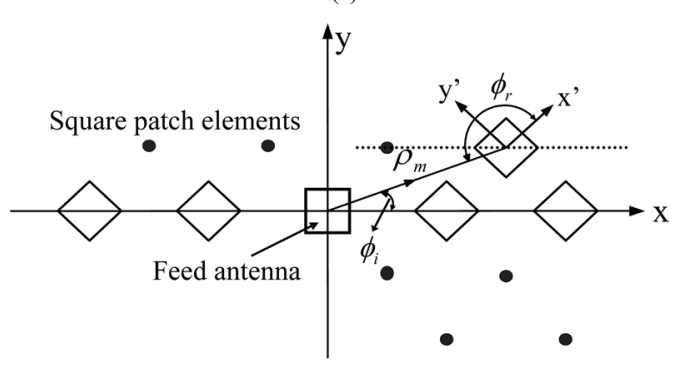

(b)

Fig. 2. Coordinates for the analysis of the folded reflectarray antenna.

phase and tapered amplitude distribution, with polarization perpendicular to the grid lines. Thus, the reradiated fields could penetrate through the subreflector and generate a narrow radiation beam.

The beam direction will vary with the position of the feed. Different from the single mode horn-fed multiple-beam reflectarray antennas which have already been extensively discussed [11], [12], three feed patches were designed and implemented in the vicinity of the center of the main reflector for radar mode beam switching in our work.

On the other hand, the feed for the communication mode is designed to have a polarization perpendicular to the grid lines on the subreflector. Therefore, the fields radiated from this feed will transmit through the subreflector directly, the gain pattern is in principle that of the feed antenna, and the position of the feed antenna is not restricted to areas in the vicinity of the center of the main reflector.

Refer to Fig. 2. The input power fed to the feed antenna is denoted as $P_{i} . G_{f}$ and $G_{\mathrm{sq}}$ are the maximum gains of the feed antenna and the square patch antenna, respectively. The antenna gains at the direction of $(\theta, \phi)$ are thus correspondingly equal to $G_{f} \cdot g_{f}(\theta, \phi)$ and $G_{\mathrm{sq}} \cdot g_{\mathrm{sq}}(\theta \phi)$, with $g_{f}(\theta, \phi)$ and $g_{\mathrm{sq}}(\theta, \phi)$ being the normalized power pattern of the feed antenna and the square patch antenna respectively. It is noted that, the typical pattern $g_{f}(\theta, \phi)$ of the designed feed antenna and the measured $G_{f}$ of realized antenna was used in the simulations.

The power $P_{m}$ received by the $m$ th square patch can be calculated from the Friis's formula and written as

$$
P_{m}=P_{i} G_{f} G_{\mathrm{sq}} C_{m}
$$

with $C_{m}$ defined as

$$
C_{m}=\frac{g_{f}\left(\theta_{i}, \phi_{i}\right) \cdot g_{\mathrm{sq}}\left(\theta_{i}, \phi_{i}+3 \pi / 4\right) \lambda^{2}}{\left(4 \pi r_{m}\right)^{2}}
$$

here, $r_{m}\left(=\rho_{m}^{2}+4 H^{2}\right)^{1 / 2}$ is the path length from the $m$ th square patch to the feed antenna, with $\rho_{m}$ being the distance between the square patch and the feed antenna. For an illuminating angle of $\left(\theta_{i}, \phi_{i}\right)$ from the feed antenna, the receiving angle for the $m$ th square patch is $\left(\theta_{i}, \phi_{i}+3 \pi / 4\right)$.

The received power by each square patch antenna will reradiate from the same antenna. The total radiation power $P_{\text {rad }}$ at an angle of $(\theta, \phi)$ from the in-phase excited array is

$$
P_{\mathrm{rad}}(\theta, \phi)=G_{\mathrm{sq}} g_{\mathrm{sq}}(\theta, \phi) \cdot F^{2}(\theta, \phi)
$$

where the array factor $F^{2}(\theta, \phi)$ is

$$
F^{2}(\theta, \phi)=\left|\sum_{m} \sqrt{P_{m}} \cdot e^{j k_{0}\left(x_{m} \sin \theta \cos \phi+y_{m} \sin \theta \sin \phi\right)}\right|^{2}
$$

with $\left(x_{m}, y_{m}\right)$ being the global coordinates of the $m$ th square patch antenna. Thus, the total antenna gain pattern $G_{\text {total }}$ of the folded reflectarray antenna can be derived as

$$
G_{\text {total }}(\theta, \phi)=P_{\mathrm{rad}} / P_{i}=G_{f} \cdot G_{\mathrm{sq}}^{2} \cdot f^{2}(\theta, \phi)
$$

where the normalized array factor is

$$
\begin{aligned}
& f^{2}(\theta, \phi) \\
& \quad=g_{\mathrm{sq}}(\theta, \phi)\left|\sum_{m} \sqrt{C_{m}} \cdot e^{j k_{0}\left(x_{m} \sin \theta \cos \phi+y_{m} \sin \theta \sin \phi\right)}\right|^{2} .
\end{aligned}
$$

\section{DESIGN}

In this paper, we developed a $38.5 \mathrm{GHz}$ dual-mode folded reflectarray antenna. For radar mode beam switching operation, three fixed-position patch antennas were created as the feeds for three corresponding spot beams. Whereas, there is another patch for the communication mode. The details for the design of each part are presented in the following subsections.

\section{A. Feed Antenna}

As a low profile folded reflectarray, feed antennas with wider beams are preferred in order to illuminate more square patch antennas. Besides, every feed's area on the main reflector has to be small to enable multiple closely spaced beams [13]. Horn antennas are used as the feeds in general reflectarrays. A horn with sophisticated illumination design [14] would make the reflectarray have optimal aperture efficiency, but it suffers from bulky configuration and mechanical complexity. On the contrary, printed type antennas, which can be directly made on the main reflector, are advantageous to multiple-feed or movable feed design. Also, the transceiver circuits can be easily integrated on the backside of the antenna. Hence the feeds in this work are probe-fed microstrip patch antennas.

The feed patches of the folded reflectarray, having sizes $(L \times$ $W$ ) of $2.3 \times 2.4 \mathrm{~mm}^{2}$, were designed on a Duroid 5880 substrate with thickness $h$ of $0.508 \mathrm{~mm}$ and $\epsilon_{r}=2.2$. The measured return loss of the fabricated antenna (Fig. 11) shows a 10-dB bandwidth of $9 \%$ (from 36.5 to $39.9 \mathrm{GHz}$ ), with a peak value of $24.8 \mathrm{~dB}$. The broadside gain $\left(G_{f}\right)$ is $6.3 \mathrm{dBi}$, and the measured 


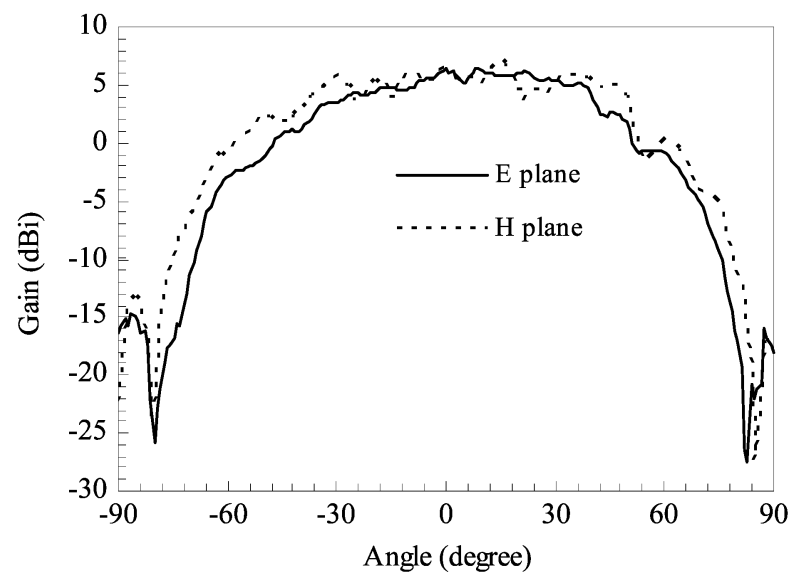

Fig. 3. The measured radiation patterns of the feed antenna at $38.5 \mathrm{GHz}$.

3-dB beamwidths in E-plane and $\mathrm{H}$-plane at $38.5 \mathrm{GHz}$ are $74.7^{\circ}$ and $88.2^{\circ}$, respectively, as shown in Fig. 3.

Several feed antennas, for both the radar and communication modes, will be jammed into between the dense square patch antennas on the main reflector. The close adjacency of the feed antennas and their neighboring square patch antennas will cause the depolarization effect on the feed antennas. The cross-polarization is lower than the co-polarization by at least $15 \mathrm{~dB}$ in simulation, which is ignorable. However, the worst isolation is only about $5 \mathrm{~dB}$ by measurement. Simple treatments around periphery of the designed feed would be required to prevent the couplings, in case that the radiation pattern for communication mode is worsen, as will be discussed in Section IV. Whereas, in the radar mode, the narrow beam patterns after focusing will scarcely affected by the variations caused by the couplings as shown in Section III $C$.

\section{B. Subreflector}

Though in general, the substrate thickness of about $\lambda / 2$ would allow the fields to go through the dielectric slab with minimum insertion losses [14]. The 31-mil Duroid 5870 laminate is chosen to fabricate the subreflector, with fine parallel metal lines printed on, because it has a low loss tangent and its relative permittivity is close to 1 . The insertion loss of a high permittivity substrate is very sensitive to the thickness. On the other hand, for low permittivity material, for example Duroid $5870, \lambda / 2$ at $38.5 \mathrm{GHz}$ is around $100 \mathrm{mil}$. It is unpractical to use such a thick laminate, despite the simulated insertion loss for this case is $0.12 \mathrm{~dB}$. The simulated loss for the 31-mil Duroid 5870 substrate at $38.5 \mathrm{GHz}$ is $0.51 \mathrm{~dB}$, which is a quite good value that can be obtained with commonly available laminates.

The width and spacing of the metal lines are designed so that the insertion loss is as low (high) as possible for an incident wave with its polarization perpendicular (parallel) to the metal lines. Three subreflectors with different printed lines were tested. The lines' width and spacing for each subreflector are kept the same, and are equal to $0.1,0.2$, and $0.5 \mathrm{~mm}$, respectively. A horn antenna illuminated EM waves toward another horn antenna at $230 \mathrm{~mm}$ apart. These two horns were collimated and aligned with each other and were vertically polarized.
The subreflectors were placed in the middle of the transmitting and receiving horns with metal lines oriented horizontally. The powers received by the receiving horn antenna were then compared to that without subreflector inserted. For the 0.1-mm subreflector, the insertion loss due to the presence of the subreflector is only $0.67 \mathrm{~dB}$. Then the subreflector was rotated to make the metal lines oriented to vertical direction, the power received with the subreflector is $38.49 \mathrm{~dB}$ lower than that without the subreflector. It is evident that the incident wave parallel to the metal lines is almost totally blocked and reflected by the subreflector.

Note that the nonzero insertion loss represents a certain portion of the incident power will be reflected. Those waves will bounce between the main reflector and the subreflector, experience multireflection and polarization twisting, and thus would impair the antenna performance.

Although not presented here, the measurement results for the $0.2-\mathrm{mm}$ and $0.5-\mathrm{mm}$ subreflectors show that the blocking effect for the parallel incident fields becomes worse when the line width and spacing increases. Thus, the $0.1-\mathrm{mm}$ subreflector was adopted for use in the folded reflectarray.

\section{Main Reflector}

The efficiency of a reflectarray is usually not that high as compared to a conventional reflector antenna. The reduction in efficiency mainly results from the power losses in the stubs-attached patch elements and the phase and polarization errors due to mutual coupling between nonidentical elements. Although the typical values for reflectarrays range from $10 \%$ to $30 \%$, efficiency up to $70 \%$ has been reported [15]. The aperture efficiency $(\eta)$ is defined as

$$
\eta=\frac{G_{\max }}{\left(4 \pi / \lambda^{2}\right) \cdot A_{p}}=\frac{G_{\max }}{(\pi D / \lambda)^{2}}
$$

here, $A_{p}\left(=\pi D^{2} / 4\right)$ is the area of the main reflector and $G_{\max }$ is the maximum antenna gain.

To get a maximum density of the array elements, the square patches are arranged to form an equilateral triangular array with spacing $S$ equal to $5 \mathrm{~mm}$, or about $0.6 \lambda$, within a circular area with diameter $D$. In general, the aperture efficiency for a small reflectarray, even with reasonable good illumination, is not very good, but gets much better with increasing size and adapted illumination.

Fig. 4 shows the calculated aperture efficiency of the folded reflectarray antenna as a function of $D$ when illuminated by the same feed patch. As the antenna size increases, the efficiency first increases due to the fast growth of the antenna gain. Then, when $D$ is further increased, the efficiency slightly decreases. This is because that the illumination power decreased as the distance and angle from the feed patch increases; the received power of the square patch antenna decays rapidly as the patch moves far away from the feed antenna, and thus the gain of the whole reflectarray becomes saturated. A maximum calculated efficiency of about $25 \%$ could be achieved as $D$ equals $140 \mathrm{~mm}$. However, when $D$ becomes larger than $100 \mathrm{~mm}$, the efficiency growth by enlarging the array size is quite limited, since the normalized received powers of the square patches located outside 


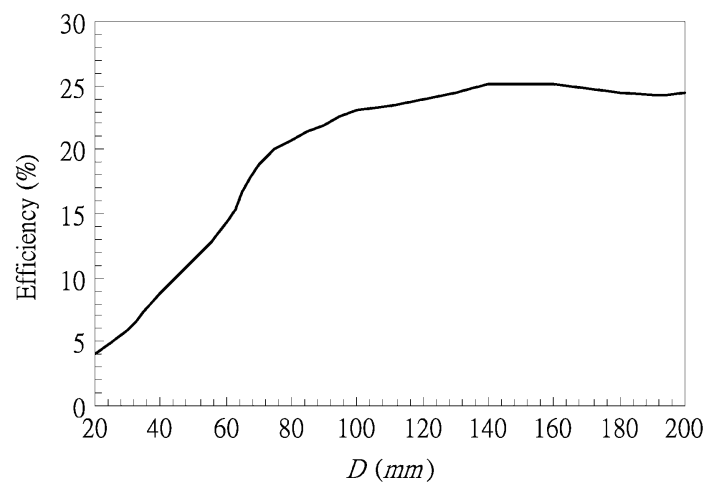

Fig. 4. Calculated aperture efficiency, as a function of the aperture's diameter $\mathrm{D}$ of the folded reflectarray antenna.

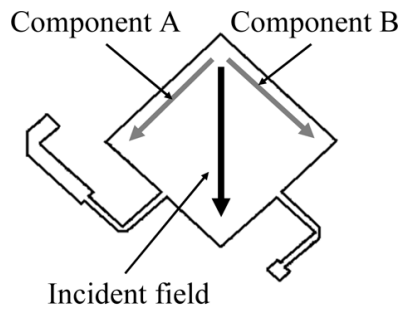

(a)

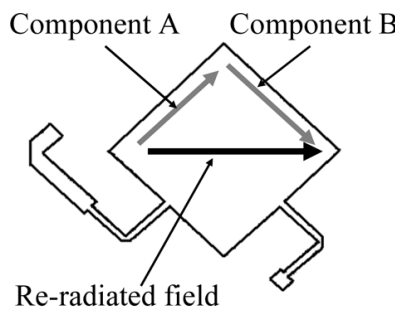

(b)
Fig. 5. The field twisting effect of the $45^{\circ}$ tilted square patch antenna with two open stubs.

the circular region of radius $50 \mathrm{~mm}$ are below $-8.3 \mathrm{~dB}$. Therefore, $D$ is determined to be $100 \mathrm{~mm}$ in this work.

The same substrate material as that for the feed antenna was used to fabricate the main reflector. The main reflector comprises several hundreds of square patch antennas located inside a circular area of diameter $D=100 \mathrm{~mm}$. The square patch antennas are oblique to the feed antenna with $45^{\circ}$. For field twisting and focusing, two microstrip open stubs, each with a $\lambda / 4$ impedance transformer, are attached to two adjacent edges of the square patch, as shown in Fig. 5. The square patch measures $2.3 \times 2.3 \mathrm{~mm}^{2}$. The widths of the stubs and the transformers are, respectively, 0.17 and $0.1 \mathrm{~mm}$. The field incident on the antenna will be received by the two stubs, reflected at the open end, and then fed back to the antenna for reradiation. The difference between the open stubs' lengths is designed to be $\lambda / 4$. This will make the reradiated field orthogonal to the incident field, as will be explained in the next paragraph. Besides, the absolute lengths of the stubs are determined according to the location of each square patch so as to compensate the phase delay due to path differences. Therefore, the antennas on the main reflector are excited with uniform phase and tapered amplitude distribution.

To see the field twisting effect, let us consider a vertical field (radiated from the feed antenna) incident on the stubs-attached patch. As shown in Fig. 5(a), the incident field can be decomposed into two orthogonal components, that is, component $\mathrm{A}$ and component $\mathrm{B}$. These two equal-amplitude components are separately received by the two orthogonal open stubs on the left and right sides. After reflected at the stubs' open ends, these components are fed back to the antenna. Since the left stub is

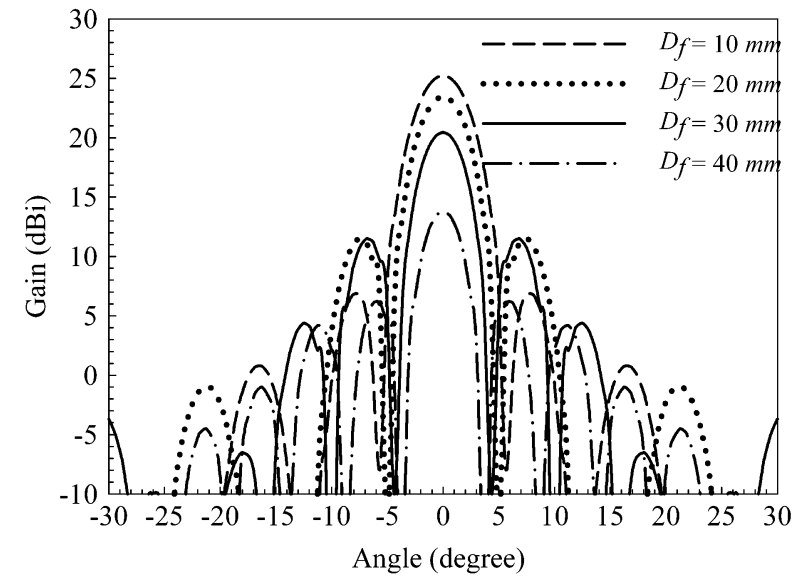

Fig. 6. Calculated H-plane patterns of the folded reflectarray antenna for various $D_{f}$ at $38.5 \mathrm{GHz}$.

longer than the right stub by $\lambda / 4$, component $\mathrm{A}$ experiences $180^{\circ}$ more phase delay than component $\mathrm{B}$ in the round-trip tour. The resultant total reradiation field is thus twisted to the horizontal direction, as shown Fig. 5(b).

For better estimation of the folded reflectarray's performance, scattering parameters of the individual square patch antenna was measured by extending the two open stubs as two ports. Around the design frequency of $38.5 \mathrm{GHz}$, the return loss $\left(\mathrm{S}_{11}\right)$ and isolation between ports $\left(\mathrm{S}_{21}\right)$ are less than $-20 \mathrm{~dB}$. Also, the co-polarization and cross-polarization patterns were taken with one port terminated. The broadside gain and the 3 -dB beamwidth of the co-polarization component are $5.6 \mathrm{dBi}$ and $88^{\circ}$ respectively. By putting the measured patterns of the individual square patch into (5), a co-polarization gain of about $26 \mathrm{dBi}$ and a maximum cross-polarization gain (assumed the cross-polarization fields of the square patches were in-phase) of $-4.8 \mathrm{dBi}$ of the folded reflectarray were obtained.

Furthermore, derived from the full wave simulation result of the single square patch with appropriate periodic boundary conditions applied, the maximum cross-polarization of the folded reflectarray grows up from $-4.8 \mathrm{dBi}$ to about $-1.5 \mathrm{dBi}$. It is the mutual coupling effect by the close proximity of the square patches and the stubs. Actually, the cross-polarization component of the array is at least $27.5(=26(-1.5)) \mathrm{dB}$ lower than the co-polarization component of the array, which is very small and will be blocked by the subreflector.

For more efficient use of the excitation power and suppressing the pattern ripple, the feed antennas for radar mode should be placed as near the center of the array as possible. Square patch antennas overlap the feed antennas are to be detached for the accommodation of the feed antennas. Therefore, since the effective aperture reduces, the main beam gains will decrease and the side lobes will increase. Assume that there are no square patch antennas within the circular area of diameter $D_{f}$. The simulated patterns for various $D_{f}$ are shown in Fig. 6 . When $D_{f}=30$ $\mathrm{mm}$, the gain drops about $1.27 \mathrm{~dB}$ in comparison to $D_{f}=0$ $\mathrm{mm}$. In reality, the feeds will occupy an area substantially less than that of the $D_{f}=30 \mathrm{~mm}$ circle. The pattern will not be significantly affected. 


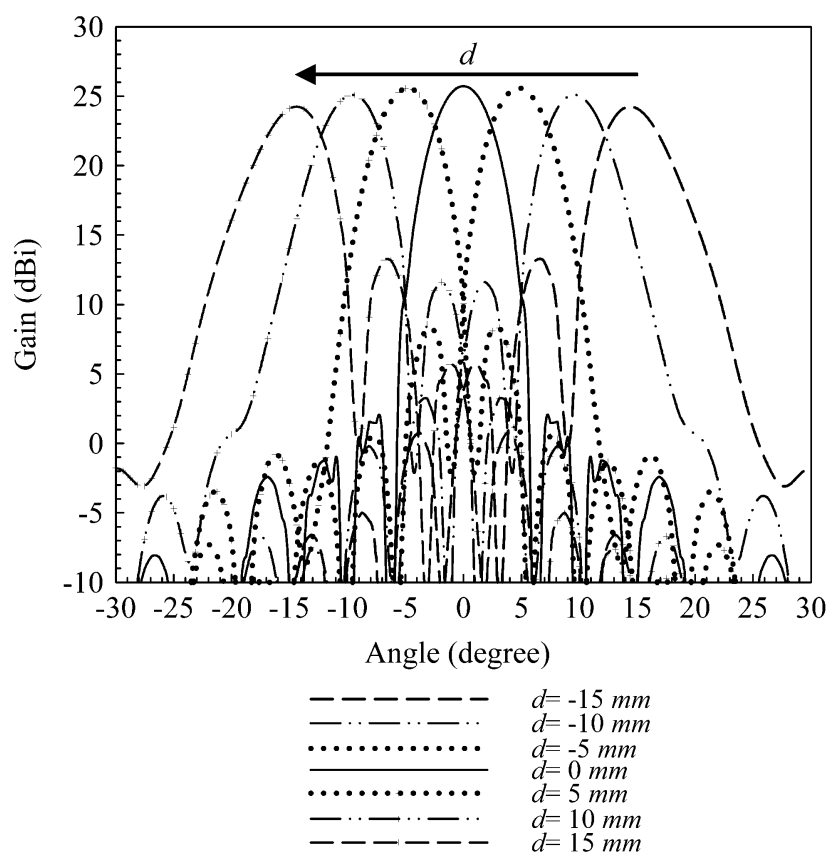

Fig. 7. Calculated H-plane patterns of the folded reflectarray antenna for various displacements (d) of the feed position at $38.5 \mathrm{GHz}$.

\section{Beam Switching Mechanism}

As can be seen from Fig. 2 that, by moving the position of the feed antenna, the path length from the image feed antenna to the $m$ th square patch changes from the original value $r_{m}$ to a new value $r_{m^{\prime}}$, and then the new normalized array factor becomes

$$
\begin{aligned}
f^{2}(\theta, \phi)=g_{\mathrm{sq}}(\theta, \phi) & \mid \sum_{m} \sqrt{C_{m}} \\
\cdot & \left.e^{j k_{0}\left(x_{m} \sin \theta \cos \phi+y_{m} \sin \theta \sin \phi+\Delta r_{m}\right)}\right|^{2}
\end{aligned}
$$

the path length variation for a small feed movement has negligible influence on the received power $\left(P_{m}\right)$, but the shift $k_{0} \Delta r_{m}\left(=k_{0}\left(r_{m}-r_{m^{\prime}}\right)\right)$ in the phase term of the antenna gain pattern would result in a beam direction change.

Fig. 7 shows the calculated H-plane gain patterns for various displacements $d$ of the feed position. The feed antenna moves along the $\mathrm{x}$ direction from $d=-15 \mathrm{~mm}$ to $+15 \mathrm{~mm}$. When $d=0 \mathrm{~mm}$, the radiation beam points to the broadside direction, with the antenna gain of $25.73 \mathrm{dBi}, 3-\mathrm{dB}$ beamwidth of $4.9^{\circ}$, and side lobe levels of about $-25 \mathrm{~dB}$. It is seen that, as $d$ changes from $-15 \mathrm{~mm}$ to $+15 \mathrm{~mm}$, the main beam directs from $+14.4^{\circ}$ to $-14.4^{\circ}$, the corresponding first side lobe moves from the left hand side of the main beam to the right hand side, and the side lobe level rises with the increase of the feed position displacement. The beam steering rate is about $1^{\circ}$ per $1 \mathrm{~mm}$ movement. However, the antenna gain becomes lower as the feed moves farther away from the center position. The maximum gain variation in the range of $d=-15 \mathrm{~mm}$ to $+15 \mathrm{~mm}$ is about $1.5 \mathrm{~dB}$.

A sliding track mechanism, as shown in Fig. 8, which allows controlling the movement of the feed, was fabricated to verify the above analysis. The center region of the main reflector was

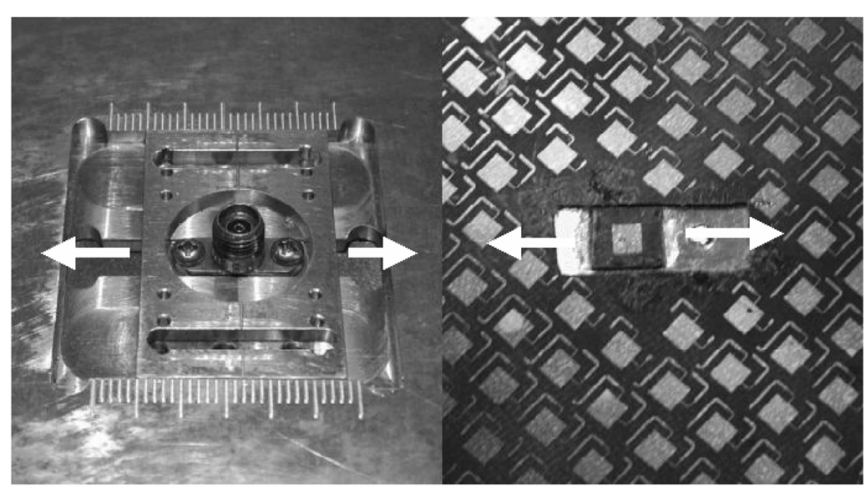

Fig. 8. Bottom view (left) and top view (right) of the sliding track mechanism, which is mounted with the main reflector.

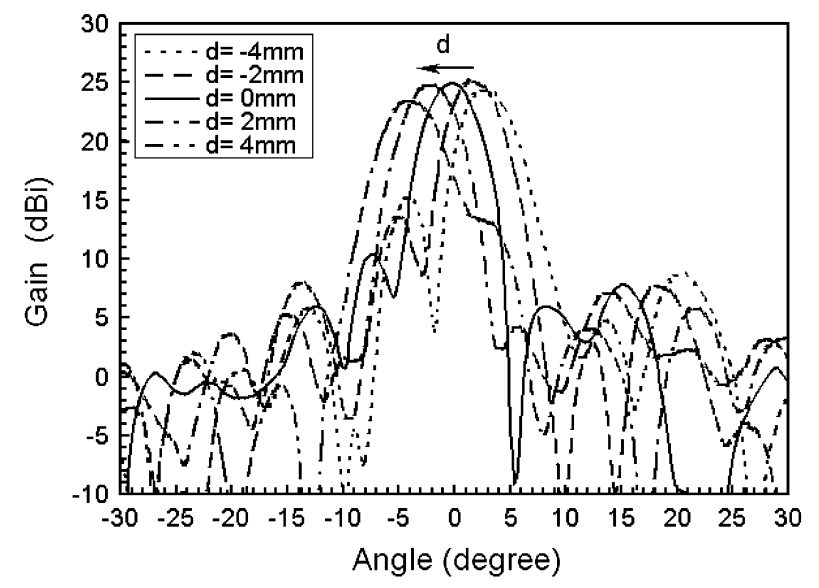

Fig. 9. The measured H-plane patterns for various feed positions (d) at $38.5 \mathrm{GHz}$.

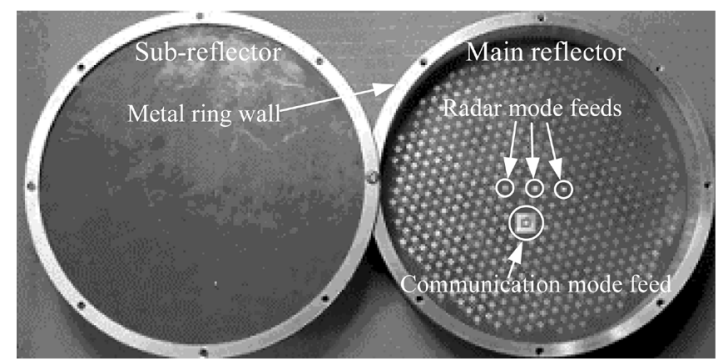

Fig. 10. Photo of the finished folded reflectarray antenna.

dug out so that the feed antenna can be placed and moved. The total movable range is $8 \mathrm{~mm}(d=-4 \mathrm{~mm}$ to $+4 \mathrm{~mm})$. The measured $\mathrm{H}$-plane patterns of the folded reflectarray antenna for various feed positions $(d)$ at $38.5 \mathrm{GHz}$ are illustrated in Fig. 9. A total steering angle of $7.2^{\circ}$ was attained for the $8-\mathrm{mm}$ feed position displacement. The beam steering rate is very close to the simulated one.

\section{RESULTS}

Fig. 10 shows the photo of the finished folded reflectarray antenna with the subreflector uncovered. An aluminum ring wall with inner diameter of $128 \mathrm{~mm}$ and height of $23 \mathrm{~mm}$ was used as the housing to support and separate the two reflectors. The three feeds for the radar mode are lined up along the $\mathrm{x}$ direction, with 


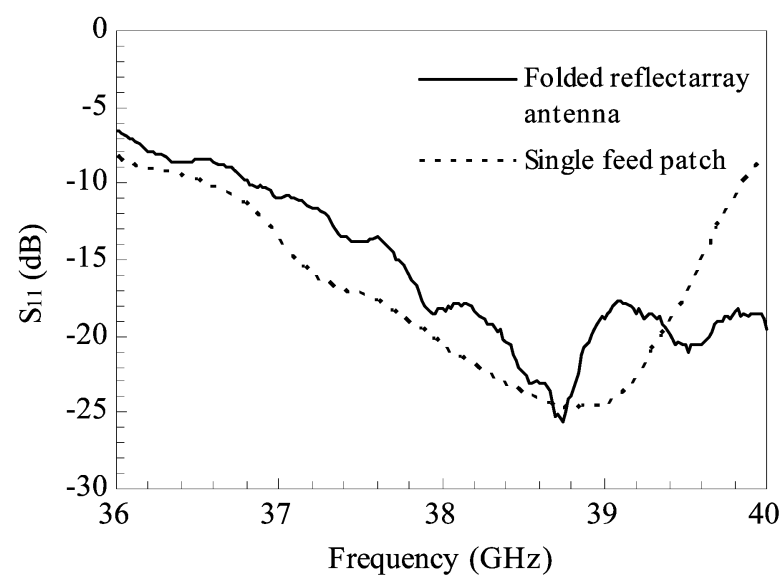

Fig. 11. The measured return losses of the fabricated folded reflectarray antenna for $\mathrm{d}=0 \mathrm{~mm}$ feed in the radar mode, and the single feed patch.

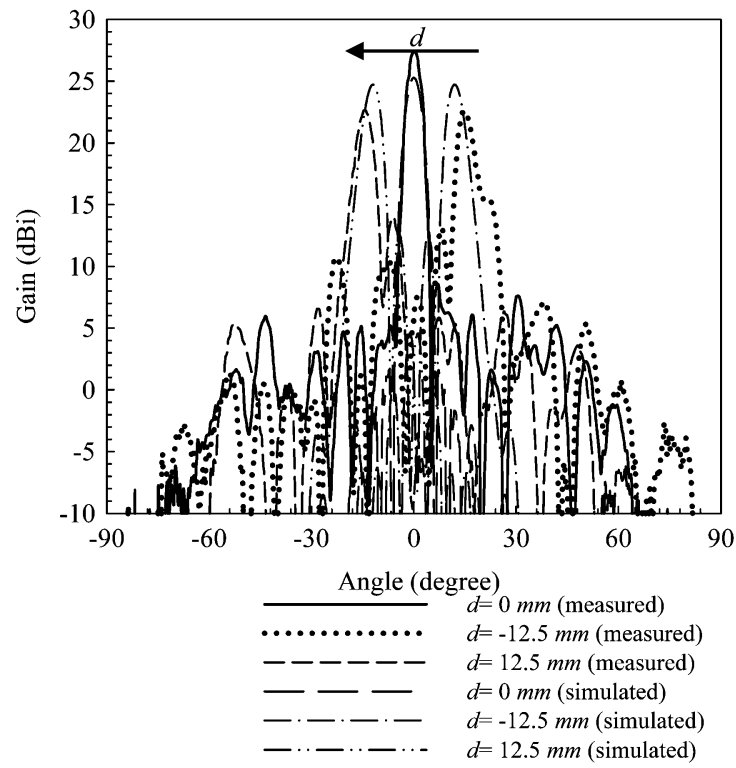

Fig. 12. The radar mode H-plane patterns of the folded reflectarray antenna at $38.5 \mathrm{GHz}$ for various feed positions (d).

the intervals being $12.5 \mathrm{~mm}$, and located at the center of the main reflector. While the feed for the communication mode is not on the same line as the radar mode feeds and away from the center by about $14.2 \mathrm{~mm}$. The measurement results for both modes are presented in the following subsections.

\section{A. Radar Mode}

The measured return loss of the finished folded reflectarray for $d=0 \mathrm{~mm}$ feed is shown in Fig. 11. It is $-22.4 \mathrm{~dB}$ at $38.5 \mathrm{GHz}$. Fig. 12 illustrates the measured $\mathrm{H}$-plane patterns of the folded reflectarray antenna for various feed positions $(d)$ at $38.5 \mathrm{GHz}$. Simulation results are also shown for comparison. It is seen that, as $d$ changes from $-12.5 \mathrm{~mm}$ to $+12.5 \mathrm{~mm}$, the main beam directs from $+14.9^{\circ}$ to $-14.6^{\circ}$. The beam steering rate is a little larger than $1^{\circ}$ per $1 \mathrm{~mm}$ movement, while the simulated main beam directions are $+11.875^{\circ}$ and $-11.875^{\circ}$ for $d=-12.5 \mathrm{~mm}$ and $d=+12.5 \mathrm{~mm}$, respectively. The measured gains of the antenna for $d=-12.5 \mathrm{~mm}, d=0$ $\mathrm{mm}$, and $d=+12.5 \mathrm{~mm}$ are $22.5,27.4$, and $22.6 \mathrm{dBi}$, respectively. The maximum aperture efficiency is about $33.9 \%$, which is better than the simulated one of $23.0 \%$. The measured $3-\mathrm{dB}$ beamwidths are $4.9^{\circ}$ in the E-plane and $4.4^{\circ}$ in the H-plane. Besides, the measured side lobe levels are $-15.5 \mathrm{~dB}$ in the E-plane and $-18.8 \mathrm{~dB}$ in the H-plane.

The measurement results agree well with the simulations in the main beam region, yet behave worse in the side lobe areas owing to the blockage of the feed antennas and the phase errors caused by mutual couplings between the square patches. Also, parts of the waves reflected at the grid lines are rereflected at the main reflector and spreading around in the antenna, leading to increased side lobes.

In fact, the beam scanning angle introduced by placing the feed off the focal point decreases with focal length to diameter ratio for a reflector antenna. Nonlinear phase as a function of feed displacement leads to pattern distortion, including beam broadening, rise in side lobe levels, and gain loss. These effects worsen with increasing feed displacement for a reflector antenna with a short focal length like this [16].

\section{B. Communication Mode}

The communication mode is expected to have a pattern resembles that of the feed patch. Unfortunately, the measured pattern exhibits a high ripple level. The major reason would be that, on the main reflector, the square patch elements in the close proximity of the communication mode feed patch would be excited by the couplings from that feed, and the radiations from those excited square patch elements would spoil the pattern. In addition, the cross-polarization component of the communication mode feed operates in the radar mode, which will be reflected by the subreflector and then reradiated from the square patches, so that the pattern will be interfered. The introduction of the subreflector would be another influential factor.

In order to ameliorate the ripple level, the subreflector was first removed, and then the effects of various treatments on the feed patch for the communication mode were investigated. The feed patch was enclosed by metal traces with different shapes and widths. Some of the most efficient methods are illustrated in Fig. 13. It is obvious that, with the metal traces, the patterns have been considerably improved, as shown in Fig. 14. The case that the feed patch surrounded by a metal square frame [Fig. 13(d)] was selected and implemented, because its pattern is most similar to that of a typical patch antenna and has the most mitigated fluctuations. The co-polarization pattern becomes smoother and more symmetric, and the gain is slightly increased. This decision is also corroborated by the fact that the measured cross-polarization fields are at least $10 \mathrm{~dB}$ lower than that without the metal square frame, as shown in Fig. 15. The measured return losses of the finished folded reflectarray for various feed antennas presented in Fig. 13 at $38.5 \mathrm{GHz}$ are shown in Fig. 16. The return loss at $38.5 \mathrm{GHz}$ for the case with Fig. 13(d) as the feed is $-21.4 \mathrm{~dB}$.

Then, the subreflector was reinstalled while keeping the square frame enclosed patch [see Fig. 13(d)] as the feed. The measured pattern is shown in Fig. 17. The pattern of using the original patch as the feed [see Fig. 13(a)], with the subreflector installed, is also shown for comparison. It can be seen that the 


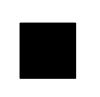

(a)

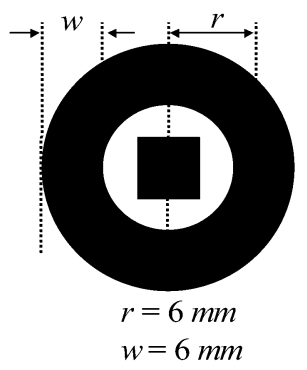

(c)

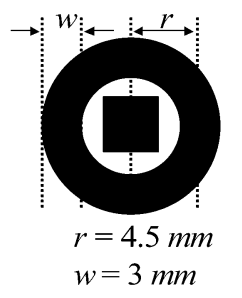

(b)

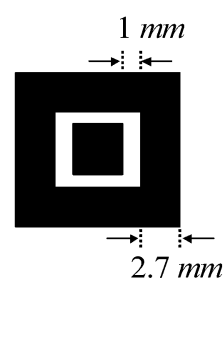

(d)
Fig. 13. Four communication mode feed antennas: (a) The original feed patch described in Section III. (b) The original feed patch enclosed with a metal ring trace. The width of the trace (w) is $3 \mathrm{~mm}$ and the radius of the ring (r) is 4.5 $\mathrm{mm}$. (c) The original feed patch enclosed with a larger metal ring trace. Both the width of the trace $(\mathrm{w})$ and the radius of the ring $(\mathrm{r})$ are $6 \mathrm{~mm}$. (d) The original feed patch enclosed by a metal square frame, with the trace width being $2.7 \mathrm{~mm}$ and the gap being $1 \mathrm{~mm}$.

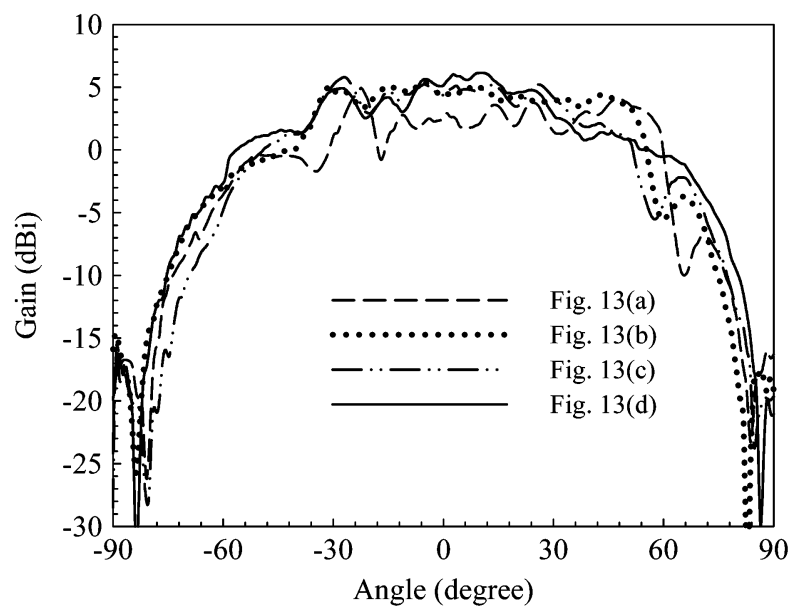

Fig. 14. The measured co-polarization patterns for various feed antennas presented in Fig. 13, with the subreflector removed.

modification made to the feed has alleviated the sharp dips, and raised the averaged gain by about $2 \mathrm{~dB}$, especially near the broadside direction (e.g., $-30^{\circ}$ to $+30^{\circ}$ ). Although the ripples are not thoroughly removed, this pattern performs satisfactorily for the inter-vehicle communications, which is an application of short-range wireless technology.

Comparing Fig. 14 with Fig. 17, it is found that the subreflector has some effect on the radiation pattern. The nonuniform phase delays experienced when the transmission fields radiated from the feed passing through the subreflector result in side lobes in the pattern because the design of the subreflector was done on the basis of normal incidence of a plane wave. The ripple level could be further improved once the transmission phase characteristics of oblique incident waves with var-

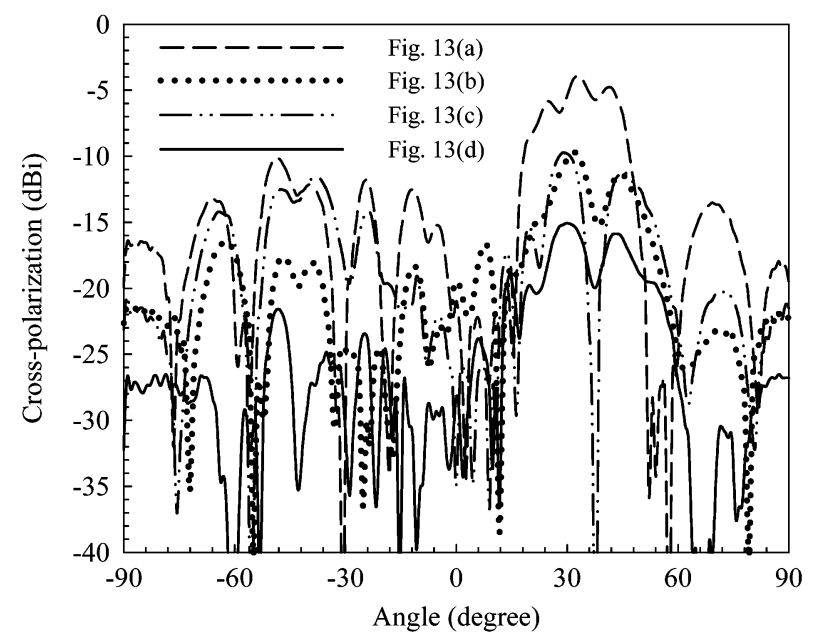

Fig. 15. The measured cross-polarization patterns for various feed antennas presented in Fig. 13, with the subreflector removed.

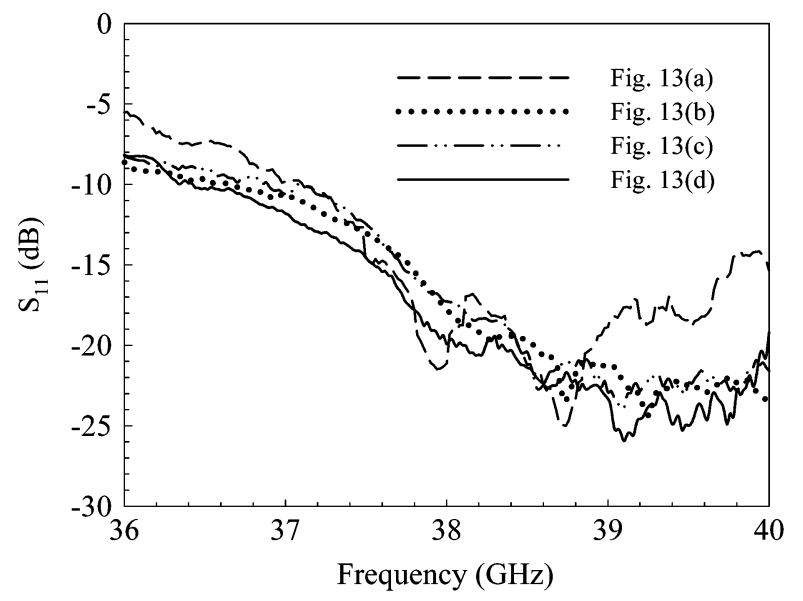

Fig. 16. The measured communication mode return losses of the fabricated folded reflectarray antenna, for various feed antennas presented in Fig. 13.

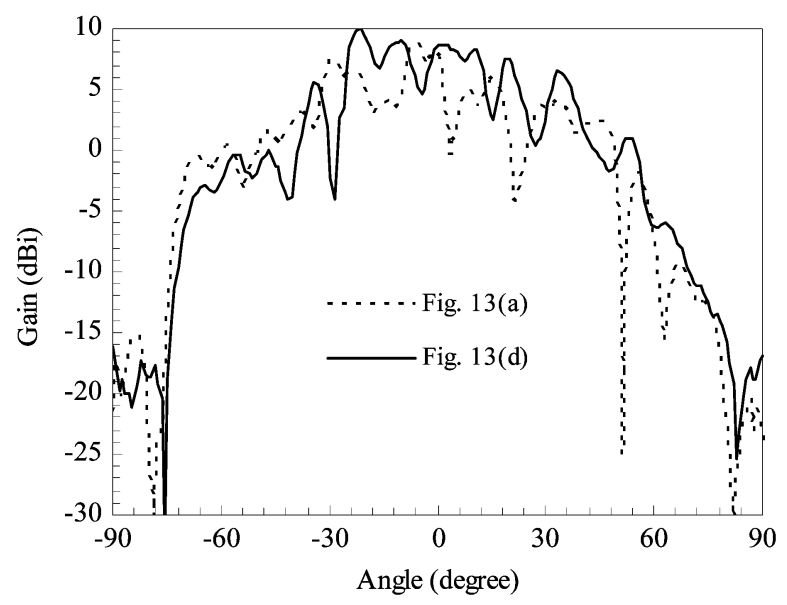

Fig. 17. The measured patterns at $38.5 \mathrm{GHz}$, with subreflector, for: (a) original feed patch [Fig. 13(a)], (b) original feed patch enclosed by a metal square frame [Fig. 13(d)].

ious angles from the feed are taken into consideration of the subreflector design. 
At last, the metal ring wall was removed and several dielectric posts were used instead to support the main reflector and subreflector. The patterns were measured and the results show that the influence of the metal wall is insignificant.

\section{CONCLUSION}

A $38.5 \mathrm{GHz}$ dual-mode folded reflectarray antenna has been proposed and demonstrated. In the radar mode, the radiation beam can be steered by switching between feeds. Three feed patches for the radar mode were implemented at different positions of the main reflector. The total beam switching angle is $29.5^{\circ}$. At the design frequency, the finished folded reflectarray antenna possesses an antenna gain of larger than $20 \mathrm{dBi}$ within the beam switching range. A maximum gain of $27.4 \mathrm{dBi}$ and the corresponding aperture efficiency of $33.9 \%$ were achieved. While for the communication mode, the ripple in the pattern was improved by enclosing the feed patch with a square metal frame. The proposed millimeter-wave folded microstrip reflectarray antenna works well for both the radar and communication mode, which is one of the likeliest candidates for an ITS antenna.

\section{REFERENCES}

[1] D. G. Barry et al., "The reflectarray antenna," IEEE Trans. Antennas Propag., vol. AP-11, pp. 645-651, Nov. 1963.

[2] D. M. Pozar, S. D. Targonski, and H. D. Syrigos, "Design of millimeter wave microstrip reflectarrays," IEEE Trans. Antennas Propag., vol. 45, no. 2, pp. 287-296, Feb. 1997.

[3] R. D. Javor, X. D. Wu, and K. Chang, "Design and Performance of Microstrip Reflectarray Antenna," IEEE Trans. Antennas Propag., vol. 43, no. 9, pp. 923-939, Sept. 1995.

[4] J. Huang, "Microstrip reflectarray," in Proc. IEEE Int. Symp. Antennas Propag., Jun. 1991, pp. 612-615.

[5] J. Huang, "Capabilities of printed reflectarray antennas," in IEEE Phased Array Systems and Technology Symp., Boston, MA, Oct. 1996, pp. 131-134.

[6] J. Huang, "Bandwidth study of microstrip reflectarray and a novel phased reflectarray concept," IEEE Trans. Antennas Propag., vol. 45, pp. 287-295, Feb. 1997.

[7] K. Y. Sze and L. Shafai, "Microstrip patches for a reflectarray," in Proc. IEEE Int. Symp. Antennas Propagation, 1999, vol. 3, pp. 1666-1669.

[8] D. Pilz and W. Menzel, "Folded reflectarray antenna," Electronics Lett., vol. 34, no. 9, pp. 832-833, Apr. 1998.

[9] W. Menzel, D. Pilz, and R. Leberer, "A $77 \mathrm{GHz}$ FM/CW radar front end with a low-profile, low-loss printed antenna," in IEEE MTT-S Digest, 1999 , pp. $1485-1488$.

[10] J. Huang, "The development of inflatable array antennas," IEEE Antennas Propag. Ma., vol. 43, no. 4, pp. 44-50, Aug. 2001.

[11] M. Thiel; and W. Menzel, "A multiple-beam sector antenna with a dual planar reflectarray arrangement," in Proc. 3rd Eur. Radar Conf., Manchester, U.K., Sep. 2006, pp. 53-56.

[12] W. Menzel, M. Al-Tikriti, and R. Leberer, "A 76 GHz multiple-beam planar reflector antenna," in Proc. Eur. Microw. Conf., Milano, Italy, Sep. 2002, vol. 3, pp. 977-980.

[13] W. Menzel, D. Pilz, and M. Al-Tikriti, "Millimeter-VVave folded reflector antennas with high gain, low loss, and low profile," IEEE Magazine Antennas Propag., vol. 44, no. 3, pp. 24-29, Jun. 2002.
[14] J. A. Zornoza, R. Leberer, J. A. Encinar, and W. Menzel, "Folded multilayer microstrip reflectarray with shaped pattern," IEEE Trans. Antennas Propag., vol. 54, no. 2, pp. 510-518, Feb. 2006.

[15] J. Huang and R. J. Pogorzelski, "Microstrip reflectarray with elements having variable rotation angles," in Proc. IEEE Int. Symp. Antennas Propagation, 1997, vol. 2, pp. 1280-1283.

[16] W. L. Stutzman and G. A. Thiele, Antenna Theory and Design.. New York: Wiley, 1997, pp. 338-342.

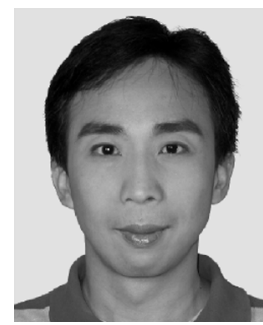

I-Young Tarn was born in Taipei, Taiwan, R.O.C. He received the B.S. and M.S. degrees in electrical engineering from Yuan-Ze University, Tao-Yuan, Taiwan, R.O.C., in 1993 and 1995, respectively, He is currently working toward the Ph.D. degree in communication engineering at the National Chiao Tung University, Hsinchu, Taiwan, R.O.C.

From 1995 to 1999, he was an Assistant Researcher on the Systems Engineering Project, National Space Program Office, Hsinchu, Taiwan, R.O.C. Since 2000, he has been with the Electrical Engineering Division of the National Space Organization (NSPO, former known as the National Space Program Office), where he has been involved in satellite communications and antenna design. His research interests include microwave/mm-wave planar antennas, reflectarray antennas, circular polarization selective structures and satellite antenna design and verification.

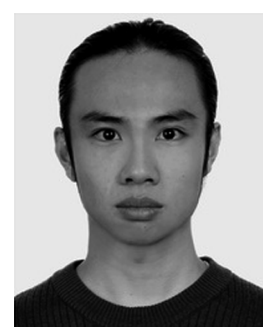

Yu-Shin Wang was born in Taichung, Taiwan, R.O.C., in May 1981 He received the B.S. degree in communication engineering from the National Chiao Tung University, Hsinchu, Taiwan, R.O.C., in 2003 , where he is currently working toward the $\mathrm{Ph} . \mathrm{D}$. degree in communication engineering.

$\mathrm{He}$ is currently involved with research on microwave circuits, antennas and antenna arrays.

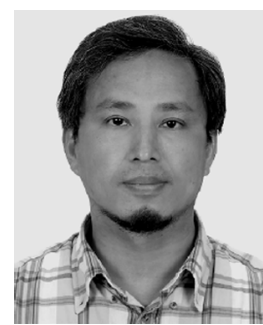

Shyh-Jong Chung (M'92-SM'06) was born in Taipei, Taiwan, R.O.C. He received the B.S.E.E. and $\mathrm{Ph} . \mathrm{D}$. degrees from National Taiwan University, Taipei, Taiwan, R.O.C., in 1984 and 1988, respectively.

Since 1988, he has been with the Department of Communication Engineering, National Chiao Tung University, Hsinchu, Taiwan, R.O.C., where he is currently a Professor. From September 1995 to August 1996, he was a Visiting Scholar with the Departmen of Electrical Engineering, Texas, A\&M University, College Station. His areas of interest include the design and applications of active and passive planar antennas, communications in intelligent transportation systems (ITSs), LTCC-based RF components and modules, packaging effects of microwave circuits, and numerical techniques in electromagnetics.

Dr. Chung received the Outstanding Electrical Engineering Professor Award of the Chinese Institute of Electrical Engineering and the Teaching Excellence Awards of National Chiao Tung University both at 2005. He served as the Treasurer of IEEE Taipei Section from 2001 to 2003 and the Chairman of IEEE MTT-S Taipei Chapter from 2005 to 2007. 